\title{
DIABETES, DOENÇAS CARDIOVASCULARES E OBESIDADE: ANÁLISE DA LEGISLAÇÃO NA ARGENTINA, NO BRASIL E NA COLÔMBIA
}

Noncommunicable diseases: analysis of the legislation in Argentina, Brazil and Colombia

${ }^{1}$ Centro de Estudos e Pesquisas de Direito Sanitário. São Paulo/SP, Brasil.

${ }^{2}$ Núcleo de Pesquisa em Direito Sanitário, Universidade de São Paulo. São Paulo/SP, Brasil.

Correspondência: Rachelle Amália Agostini Balbinot. E-mail: rachelle@usp.br.

Recebido em: 12/09/2014. Revisado em: 27/10/2014. Aprovado em: 30/10/2014. 


\section{RESUMO}

O presente artigo aborda as Doenças Crônicas Não Transmissíveis (DCNT) - diabetes, doenças cardiovasculares e obesidade -, que ganham visibilidade devido a sua magnitude no mundo. Argentina, Brasil e Colômbia são os países selecionados para verificar a existência de legislação que trate de DCNT. Dados da Organização Mundial da Saúde (OMS) denotam a necessidade de atuação dos Estados em prol da redução da prevalência das DCNT, sendo que as doenças cardiovasculares representam a maior causa de morte no mundo. As normas legais e infralegais existentes nos três países simbolizam a importância das DCNT no contexto nacional, bem como sinalizam a necessidade de atuação institucional para reverter esse quadro. A questão central posta neste momento é como alcançar a meta estabelecida pela OMS de diminuir a prevalência das DCNT em 25\% até 2025. Para tanto, é preciso contar com o esforço conjunto do poder público, das instituições governamentais e não governamentais, da sociedade e dos indivíduos. O cenário não é otimista, mas, se bem orquestrado e contando com a colaboração de todos os envolvidos, vislumbra-se possível alcançar o sucesso nessa questão de saúde pública.

\section{Palavras-chave}

Direito à Saúde; Doenças Crônicas Não Transmissíveis; Legislação Sanitária.

\section{ABSTRACT}

This study addresses Noncommunicable diseases (NCDs), which become more visible due to their worldwide magnitude. Argentina, Brazil and Colombia are the countries selected to verify the existence of legislation that deals with NCDs. Data from the World Health Organization (WHO) indicate the need for governmental action in order to reduce the prevalence of NCDs, considering that cardiovascular diseases are the leading cause of death worldwide. Statutory and regulatory provisions in the three countries symbolize the importance of NCDs in national context, as well as signal the need for institutional action to reverse this situation. The current central question is how to achieve the WHO's objective of reducing the NCDs prevalence by $25 \%$ until 2025 . To do so, we must rely on the joint efforts by the government, governmental and non-governmental institutions, society, and individuals. The scenario is not optimistic, but if well-orchestrated and including collaboration of all involved, it is possible to envision achieving success in this public health issue.

\section{Keywords}

Health Legislation; Noncommunicable Diseases; Right to Health. 


\section{Introdução}

Entre as doenças crônicas não transmissíveis (DCNT), este estudo recai sobre diabetes, doenças cardiovasculares e obesidade, especialmente em razão do grau de importância que apresentam no cenário global. Como se verá adiante, estatísticas recentes mostram que se revela necessário e apropriado enfrentar o tema - e, nesse sentido, estudar as técnicas legislativas utilizadas - a fim de prevenir e controlar essas doenças na América Latina. É considerável o impacto atribuível a essas doenças em termos de morbidade e mortalidade, bem como o impacto financeiro gerado para os indivíduos, para suas famílias e para os sistemas de saúde, enfim, para o próprio país de forma geral. Assim, trabalhar mecanismos e estratégias que visam lidar com os chamados fatores de risco das DCNT na América Latina pode contribuir de forma significativa para o enfrentamento dessa situação, bem como auxiliar na redução dessas doenças e de suas consequências, tanto nacional como globalmente.

\section{Diabetes, doenças cardiovasculares e obesidade: dados}

De acordo com a Organização Mundial da Saúde (OMS), mais de 220 milhões de pessoas no mundo têm diabetes ${ }^{1}$ e cerca de $80 \%$ das mortes atribuídas à doença ocorrem em países de baixa e média renda. Estima-se que 1,1 milhão ${ }^{2}$ de pessoas morreu em 2005 em razão da diabetes.

A doença cardiovascular ${ }^{3,4}$, por sua vez, é a causa número um de morte no mundo: anualmente, morrem mais pessoas de doenças cardiovasculares do que de qualquer outra causa. Estima-se que 17,3 milhões ${ }^{5}$ de pessoas morreram de doenças cardiovasculares em 2013, representando mais de 30\% de todos os óbitos no mundo. Países de baixa e média renda são desproporcionalmente afetados: 82\% das doenças cardiovasculares ocorrem nesses países.

Em relação à obesidade ${ }^{6}$, os dados mais recentes da OMS indicam que, em 2005, aproximadamente 1,6 bilhão de adultos com mais de 15 anos de idade tinha

\footnotetext{
${ }^{1}$ ORGANIZAÇÃO MUNDIAL DA SAÚDE (OMS). Diabetes Mellitus. Fact Sheets, n. 312, Oct. 2013. Disponível em: <http://www.who.int/mediacentre/factsheets/fs312/en/index.html>. Acesso em: 23 ago. 2014.

${ }^{2}$ A OMS projeta que as mortes por diabetes dobrarão entre 2005 e 2030. Em 2030, aproximadamente 23,6 milhões de pessoas vão morrer de doenças cardiovasculares, principalmente por doenças do coração e derrames cerebrais.

3ORGANIZAÇÃO MUNDIAL DA SAÚDE (OMS). Doenças Cardiovasculares. Fact Sheets, n. 317, Mar. 2013. Disponivel em: <http://www.who.int/mediacentre/factsheets/fs317/en/index.html>. Acesso em: 31 ago. 2014.

${ }^{4}$ As doenças cardiovasculares: afetam o coração e os vasos sanguíneos como hipertensão (pressão alta), infarto, aterosclerose etc. DICIONÁRIO do Coração - Termos e Procedimentos. Disponível em: <http://prevencao.cardiol.br/biblioteca/principal.asp\#D>. Acesso em: 06 set. 2014.

${ }^{5}$ ORGANIZAÇÃO MUNDIAL DA SAÚDE (OMS). O Dia Mundial do Coração 2013 preconiza hábitos de vida saudável desde a infância. Disponível em: <http://www.paho.org/bireme/index.php?id=219\%3Ao-dia-mundial-do-coracao-2013-preconiza-habitos-de-vida-saudavel-desde-a-infancia\&option=com_content>. Acesso: em 07 set. 2014.

${ }^{6}$ ORGANIZAÇÃO MUNDIAL DA SAÚDE (OMS). Obesity and overweight. Fact Sheets, n. 311, Aug. 2014. Disponível em: <http://www.who.int/mediacentre/factsheets/fs311/en/index.html>. Acesso em: 01 set. 2014.
} 
sobrepeso e pelo menos 400 milhões de adultos eram obesos; ; além disso, pelo menos 40 milhões de crianças com menos de cinco anos de idade estavam acima do peso em 2012. Considerados um problema de saúde pública apenas em países de alta renda, o sobrepeso e a obesidade têm aumentado dramaticamente nos países de baixa e média renda, especialmente em ambientes urbanos.

Em 63\% dos óbitos anuais decorrentes das DCNT, 14 milhões de pessoas são muito jovens (menos de 70 anos) e, por sua concentração ocorrer em países de baixa e média renda, entende-se que essas mortes poderiam ter sido evitadas. Este é o cenário atual com o qual trabalha a OMS ao propor uma ação global Plan for the Prevention and Control of NCDs 2013-2020 . A proposta é fornecer um roteiro de políticas a serem implementadas entre 2013 e 2020 pelos atores envolvidos no tema (Estados, organizações não governamentais, setor privado etc.). Esse esforço conjunto poderá ocasionar a diminuição da mortalidade prematura por DCNT.

Quando consideradas as causas das DNCT, é possível observar que os chamados fatores de risco associados ao seu desenvolvimento e consequente agravamento estão intimamente relacionados a hábitos (e estilos) de vida não saudáveis. Mesmo que os fatores de risco estejam relacionados a fenômenos mais amplos, como a urbanização, ainda assim podem ser evitados com a implementação de políticas públicas de saúde 9 .

Hoje, entende-se que o estímulo à alimentação saudável, à prática de atividade física regular e à manutenção do peso corporal adequado à estrutura individual, bem como a evitar os produtos derivados do tabaco, pode prevenir ou retardar a ocorrência de diabetes mellitus ${ }^{10}$. Da mesma forma, os fatores de risco associados a doenças cardiovasculares são: dieta inadequada, sedentarismo e consumo de produtos derivados do tabaco. No que diz respeito à obesidade, são atribuídos alguns outros fatores, incluindo uma dieta em que se ingerem alimentos altamente energéticos, ricos em gordura e açúcares (e pobres em vitaminas, minerais e outros micronutrientes) agregada a uma forte tendência ao sedentarismo - devido, inclusive, ao aumento de atividades de natureza pouco dinâmicas (novas formas de trabalho, mudança nos meios de transporte e aumento da urbanização).

\footnotetext{
${ }^{7} \mathrm{~A}$ OMS projeta que, em 2015, aproximadamente 2,3 bilhões de adultos estarão com sobrepeso e mais de 700 milhões serão obesos.

${ }^{8}$ WORLD HEALTH ORGANIZATION (WHO). Resolution WHA66.10. Global action plan for the prevention and control of noncommunicable diseases 2013-2020. Disponível em: <http://apps.who.int/iris/bitstre am/10665/94384/1/9789241506236_eng.pdf?ua=1>. Acesso em: 08 set. 2014.

${ }^{9} \mathrm{BUCCl}, \mathrm{M}$. P. Dallari. O conceito de política pública em direito. In: BUCCI, M. P. Dallari (Org.). Políticas públicas: reflexões para um conceito jurídico. São Paulo: Saraiva; Santos/SP: UNISANTOS, 2006.

${ }^{10} \mathrm{Com}$ relação especificamente à prevenção do diabetes e de suas complicações, medidas relacionadas ao estilo de vida relativamente simples já mostraram ser efetivas: alcançar e manter um peso corporal saudável; ser fisicamente ativo (pelo menos 30 minutos de atividade regular, de intensidade moderada na maior parte dos dias); ter uma dieta saudável de três a cinco refeições com frutas e vegetais por dia e reduzir a ingestão de açúcares e gorduras saturadas.
} 
Em 2011, o periódico The Lancet publicou uma série de estudos para auxiliar a primeira reunião de alto nível da Assembleia Geral da ONU sobre doenças crônicas não transmissíveis ${ }^{11}$. A série contou com ampla participação de cientistas, pesquisadores e especialistas a fim de "apresentar estratégicas para incrementar ganhos de saúde, monitoramento e intensificação das intervenções"12 voltadas às DCNT. Entre as cinco prioridades de intervenção estão: controle do tabagismo, redução da ingestão de sal, promoção para aumentar o consumo de dietas saudáveis e a atividade física, redução do consumo de álcool e, por fim, acesso a medicamentos e tecnologias contra as DCNT. Estima-se que, adotando-se as estratégias propostas, seja possível reduzir em $2 \%$ ao ano o número de mortes decorrentes das DCNT. Entre as preocupações dos pesquisadores estava a propositura de ações que fossem custo-efetivas para os Estados; o valor estimado para a implementação das propostas foi de nove bilhões de dólares por ano, com base nos dados de 2007 e considerando-se 23 países de alta renda.

Enquanto este é o cenário mundial, na América Latina as taxas das DCNT têm aumentado nas últimas décadas devido a uma combinação de fatores - como a crescente urbanização, o aumento da expectativa de vida, a melhoria dos níveis socioeconômicos e as mudanças nos hábitos alimentares, entre outras causas - que propiciou uma transição epidemiológica. Especificamente, diabetes, doenças cardiovasculares e obesidade são responsáveis por altas taxas de mortalidade nos países latinos. Além do contexto epidemiológico, duas tendências preocupantes são a comorbidade entre as três doenças e a alta probabilidade de um aumento incremental nos casos, considerando-se o aumento da obesidade entre os adolescentes. Além de a obesidade associar-se à diabetes e às doenças cardiovasculares e poder contribuir para elas, é importante destacar que um adolescente obeso tem aumentadas as chances de ser um adulto obeso. Esse fato, por si só, reforça a importância da prevenção das DCNT, e o sucesso dessa empreitada contribuirá imensamente para a redução dos custos médicos e sociais no longo prazo ${ }^{13}$.

No entanto, é importante notar que um dos maiores desafios em relação às DCNT, especialmente a obesidade, é sua forma de representação. Ou seja, a noção predominante é de que as doenças crônicas são questões de cada indivíduo, quando na verdade são problemas orgânicos que, portanto, precisam

\footnotetext{
${ }^{11}$ CHRONIC diseases and development. High-level Meeting of the General Assembly on chronic non-communicable diseases. The Lancet. Disponivel em: <http://www.thelancet.com/series/chronic-diseases-and-development>. Acesso em: 13 set. 2014.

${ }^{12}$ BEAGLEHOLE, R. et al. Priority actions for the non-communicable disease crisis. The Lancet, v. 377, p. 1438-1447, 2011.

${ }^{13}$ MARTIN, R. The role of law in the control of obesity in England: looking at the contribution of law to a healthy food culture. Australia and New Zealand Health Policy, v. 5, n. 21, 2008. Disponível em: <http://www.ncbi. nIm.nih.gov/pmc/articles/PMC2615029/>. Acesso em: 26 out. 2014.
} 
de ações em nível macro para que possam ser realmente controladas ${ }^{14}$. Além disso, embora o desenvolvimento da pesquisa genética e de medicamentos para o tratamento sejam componentes necessários, estes podem ao mesmo tempo construir um cenário fantasioso que acaba por minimizar a importância da prevenção ${ }^{15}$. Por isso, é fundamental a implementação de estratégias que atuem tanto na recuperação (tratando dos casos atuais) como na prevenção (para reduzir casos futuros).

As estratégias de intervenção são agrupadas em duas grandes categorias: as intervenções focadas no indivíduo (ou seja, mudanças de comportamento e tratamento) e aquelas focadas na população (ou seja, mudanças nos ambientes físico e social). Entre as estratégias de prevenção verificam-se duas abordagens principais, uma voltada para pessoas que já apresentam alto risco, e outra, para a população em geral. A ideia é garantir que as estratégias específicas possam produzir melhores resultados, ou seja, alcançar de forma significativa a redução das doenças cardiovasculares ${ }^{16}$.

Para Lang, uma estratégia bem-sucedida deve contemplar aspectos dos dois tipos de intervenção. As mudanças no comportamento alimentar - como o aumento do consumo de frutas, legumes e verduras, a realização de atividade física juntamente com a redução do consumo de bebidas açucaradas, dos alimentos altamente calóricos e sem valor nutricional - são outras ações recomendadas voltadas aos indivíduos. Mas esses exemplos de mudança de atitudes individuais não serão suficientes para a transformação que se almeja. É imperiosa uma estrutura externa que vise a promover a mudança nos ambientes físico e social frequentados pelos indivíduos ${ }^{17}$.

Entre outras, está a estratégia de utilização do marketing social com a finalidade de auxiliar a população e de empoderá-la para uma melhor adaptação às práticas saudáveis, bem como garantir informações que visem promover mudanças no comportamento de modo amplo ${ }^{18}$.

\footnotetext{
${ }^{14}$ MAGNUSSONR. S. What's law got to do with it? Part I: a framework for obesity prevention. Australian and New Zealand Health Policy, v. 5, p. 1-12, 2008.

${ }^{15}$ SWINBURN B.; EGGER, G. Preventive Strategy against Weight Gain and Obesity. Obesity Reviews, v. 3, p. 289-301, 2002.

${ }^{16}$ PAN AMERICAN HEALTH ORGANIZATION. Population and individual approaches to the prevention and management of diabetes and obesity. Disponivel em: <www.paho.org/hq/index.php?option=com docman\&task=doc_view\&gid=15557\&ltemid=>. Acesso em: 26 out. 2014.; BLAIR, S. N.; DANIELS, S. R.; ECKEL, R. H.; FAIR, J. M. et al. AHA guidelines for primary prevention of cardiovascular disease and stroke: 2002 update: Consensus panel guide to comprehensive risk reduction for adult patients without coronary or other atherosclerotic vascular diseases. Circulation, v. 106, n. 3, p. 388-391, 2002.

${ }^{17}$ LANG, T.; RAYNER, G. Over coming policy cacophonyon obesity: na ecological public health framework for policymakers. Obesity Reviews, v. 8, p. 165-181, 2007.

${ }^{18}$ GORDON, R.; McDERMOTT, L.; STEAD, M.; ANGUS, K. The effective ness of social marketing interventions for health improvement: what's the evidence? Public Health, v. 120, n. 12, p. 1133-1139, 2006.
} 
Para reduzir efetivamente as DCNT, vários autores sugerem alterar o ambiente em torno da comunidade ou pessoa, tanto físico como social (hospitais, escolas e áreas de trabalho) ${ }^{19}$. Entre os exemplos, temos: incluir informação nutricional em cardápios para auxiliar os consumidores, construir calçadas para possibilitar que as pessoas possam andar mais e incentivar a compra de alimentos saudáveis (redução de preço, incentivo ao produtor) ${ }^{20}$.

Além de mudanças no ambiente em que a pessoa vive, Martin $^{21}$ propõe como uma necessidade a melhoria do acesso aos cuidados de saúde para que se possam reduzir as disparidades de saúde em geral, indicando a importância de incorporar temas de prevenção além de tratar da situação atualmente posta. Outras mudanças voltadas ao funcionamento e à estruturação do setor de saúde incluem a incorporação da prevenção na atenção primária e a implementação de um programa antitabaco, pois o ato de fumar associa-se aos piores resultados quando consideradas as pessoas com DCNT ${ }^{22,23}$.

O panorama necessita de comprometimento de cada um dos países e, mais uma vez, a OMS propõe uma atuação conjunta dos países: “em 2012, todos os países se comprometeram em reduzir $25 \%$ da mortalidade prematura decorrente das DCNT até 2025 (a meta de $25 \times 25$ )"24. Parte da estratégia é implementar um conjunto de metas voluntárias para tratar dos fatores de risco e sistemas de saúde.

A partir dessa contextualização e de uma breve revisão de literatura, buscou-se na legislação dos países latino-americanos estudados - Argentina, Brasil e Colômbia - construir um panorama legislativo sobre as DCNT.

\section{Legislação sobre prevenção e controle das DCNT na Argentina, no Brasil e na Colômbia}

A necessidade governamental de enfrentar o aumento progressivo das DCNT pode ser acompanhada por meio da legislação. O crescente número de

\footnotetext{
${ }^{19}$ JEWELL, J.; HAWKES, C.; ALLEN, K. Law and obesity prevention: addressing some key questions for the public health community. WCRF International, 2013; POMERANZ, J. L.; GOSTIN, J. D. Improving laws and legal authorities for obesity prevention and control. Journal of Law, Medicine and Ethics, n. 37, p. 62-75, 2009; SRINIVASAN, S.; O'FALLON, L. R.; DEARRY, A. Creating healthy communities, healthy homes, healthy people: research agenda on the built environment and public health. American Journal of Public Health, v. 93, n. 9, p. 1446-1450, 2003.

${ }^{20}$ MARTIN, R. op. cit.; FRIEDMAN, R. R.; SCHWARTZ, M. B. Public policy to prevent childhood obesity and the role of pediatric endocrinologists. Journal of Pediatric Endocrinology \& Metabolism, v. 21, p. 717-725, 2008; JEWELL, J.; HAWKES, C.; ALLEN, K. op. cit.

${ }^{21}$ MARTIN, R. op. cit.

${ }^{22}$ CHIOLERO, R.; FAEH, D.; PACCAUD, F.; CORNUZ, J. Consequences of smoking for body weight, body fat distribution, and insulin resistance. American Society for Clinical Nutriton, v. 87, n. 4, p. 801-809, 2008.

${ }^{23}$ CAMPOS, T. et al. Cigarette smoking among patients with chronic diseases. International Journal of Cardiology, v. 174, n. 3, p. 808-810, 2014.

${ }^{24}$ BEAGHOLE, R. et al. NCD Countdown 2025: accountability for the $25 \times 25$ NCD mortality reduction target. The Lancet, v. 384, n. 9938, p. 105-107, 2014. Disponível em: <http://www.thelancet.com/pdfs/journals/ lancet/PIIS0140673614610916.pdf>. Acesso em: 13 set. 2014.
} 
leis e sua especialização (normas específicas para diabetes, doenças cardiovasculares e obesidade) evidenciam não só o impacto que tais doenças causam nos sistemas de saúde, como também, consequentemente, a indispensabilidade de atuações institucionais.

O estudo das DCNT deve-se aos mesmos fatores de risco: hipertensão arterial, obesidade, colesterol elevado, tabagismo e baixa atividade física.

Importa notar que, a partir dos anos 2000, as DCNT começam a ganhar relevância no mundo devido ao número de mortes por elas causado. Assim, é possível afirmar que temos um movimento legislativo latino-americano caracterizado pelo aumento de normas (legais e infralegais) para tratar das DCNT. Alguns países optam por uma lei geral para tratar das DCNT e outros, por leis específicas voltadas à prevenção e recuperação de cada uma delas. Este estudo buscou mostrar a centralidade da questão das DCNT para a saúde pública, por meio da legislação atualmente vigente na Argentina, no Brasil e na Colômbia.

\section{Argentina}

Em 2008 na Argentina, 80\% das mortes foram atribuídas às doenças crônicas não transmissíveis, sendo $33 \%$ delas causadas por doenças cardiovasculares. Ainda é presente uma proporção considerável de fatores de risco na população: inatividade física $(68,99 \%)$, sobrepeso $(64,2 \%)$, obesidade $(29,7 \%)$, hipertensão arterial $(36,7 \%)$ e tabagismo diário $(23,6 \%)^{25}$.

Com o intuito de modificar esse cenário, a Argentina promulgou a Resolução n. 444/2007, criando o Programa Nacional Argentina Camina, que tem entre seus objetivos:

Art. $2^{\circ}$. O Programa Nacional Argentina Camina terá entre suas competências e funções:

(...)

c) Estabelecer a criação de uma rede de instituições que promovam ações de incentivo a um estilo de vida ativo e intercambio de experiências; (...)

j) Criar e gerir o site "Argentina Camina" contendo link para o site do Ministério da Saúde e cujos componentes mínimos incluirão:

- Informações para o público;

\footnotetext{
${ }^{25}$ ORGANIZAÇÃO PAN-AMERICANA DA SAÚDE (OPAS). Saúde nas Américas: panorama geral e perfis de países, 2012. Disponivel em: <http://apsredes.org/site2012/wp-content/uploads/2012/09/Sa\%C3\%BAde-nas-Am\%C3\%A9ricas3.pdf>. Acesso em: 28 ago. 2014.
} 
- Registro de municípios que aderiram ao Programa Argentina Camina;

- Divulgação das atividades realizadas e fortalecimento do intercâmbio de informações por meio da atualização e difusão permanente;

- Coordenação e difusão de informações com links para os municípios, agências, organizações e redes que já têm página na web sobre o mesmo assunto, tanto na Argentina como em outros países e organizações internacionais;

- Informações para profissionais;

- Ferramentas metodológicas para iniciativas nos municípios, locais de trabalho e escolas;

- Outras publicações de interesse.

k) Criar e administrar um registro de "Empresas Saudáveis" e seu respectivo sistema de acreditação.

A resolução dispõe e cria condições para mudanças não só individuais também necessárias para a redução das doenças crônicas - como ainda estimula comportamentos coletivos e sociais para que esse objetivo possa ser alcançado.

Outra Resolução, de n. 738/2008, trata do Programa Cuidarse en Salud ${ }^{26}$ e apresenta a promoção e a prevenção como importantes estratégias, que devem estar combinadas com a comunicação social e a educação para minimizar os já conhecidos fatores de risco.

O centro é a atenção primária à saúde:

(...) enfatiza a necessidade de implementar, iniciativas de saúde sustentáveis subsequentes no tempo e no espaço, em diferentes graus de amplitude e profundidade, incorporando estratégias de promoção e prevenção da saúde para informar e capacitar os cidadãos, aumentando a consciência dos direitos e a promoção de comportamentos saudáveis ${ }^{27}$.

É na atenção primária que se pode efetivamente garantir o empoderamento do indivíduo e, assim, ampliar sua capacidade de enfrentamento das doenças crônicas.

O Decreto n. 621/2008, do Poder Executivo Nacional (PEN) ${ }^{28}$, trata do Plan Nacional de Abordaje Integral e tem como objetivo “implementar políticas públicas inte-

\footnotetext{
${ }^{26}$ ARGENTINA. Ministerio de Salud. Resolución 738/2008. Disponível em: <http://test.e-legis-ar.msal.gov. ar/leisref/public/showAct.php?id=11481\&word=>. Acesso em: 19 ago. 2014.

${ }^{27}$ Id. Ibid.

${ }^{28}$ ARGENTINA. Poder Ejecutivo Naciónal. Plan Naciónal de Abordaje Integral. Disponível em: <http://test.e-legis-ar.msal.gov.ar/leisref/public/showAct.php?id=7444\&word=>. Acesso em: 18 ago. 2014.
} 
grais orientadas para o efetivo cumprimento dos direitos sociais, vinculados com educação, saúde comunitária, trabalho, moradia, cultura, lazer e acesso a serviços públicos essenciais". Este decreto é bastante minucioso e lista uma série de linhas de ação preventiva.

Ainda, um importante ponto é a centralidade da participação da comunidade, como necessária para a efetiva implementação das políticas públicas de saúde:

8.1. Realização de atividades destinadas ao fortalecimento de espaços de participação cidadã, geração de cultura democrática e organização social;

8.2. Fornecimento de ferramentas teóricas e práticas para o fortalecimento das iniciativas de organização da comunidade local; e

8.3. Desenho e implementação de atividades conjuntas e tarefas de abordagem que otimizem os recursos e as potencialidades das instituições e dos atores locais.

Assim, delineia-se a construção de uma política pública de saúde voltada à prevenção das DCNT, qual seja o Plan Nacional Argentina Saludable, disposto na Resolução n. 1083/2009 (MS) ${ }^{29}$. Esse plano apresenta estratégias que estão presentes nas resoluções anteriores, e é bastante específico e detalhado.

Entre seus objetivos estão: (i) reduzir a prevalência de fatores de risco das doenças não transmissíveis na população; (ii) reduzir a mortalidade por doenças crônicas não transmissíveis; e (iii) melhorar o acesso e a qualidade do atendimento, tanto na deteç̧ão como no tratamento das pessoas em risco e afetadas por doença crônica.

Esse mesmo plano dispõe sobre a necessária articulação com os demais programas já existentes, que são intrinsecamente conectados: Programa Nacional de Prevenção e Controle de Diabetes Mellitus; Programa Nacional de Controle de Tabagismo; Programa Nacional de Controle de Câncer Genital e Mamário; Programa Nacional de Controle de Lesões ${ }^{30}$.

O elemento comum em todas as normas argentinas que tratam especialmente da prevenção das DCNT é a necessidade de participação comunitária, intersetorial e intergovernamental. São requisitos imprescindíveis para se alcançar o objetivo de reduzir a mortalidade em razão desse grupo de doenças. Para tanto, é preciso conter o aumento do número de indivíduos expostos ao risco, bem como tratar dos que já apresentam qualquer das três enfermidades crônicas. E, para que tal objetivo seja levado a cabo, a condição sine qua non é o trabalho em conjunto, institucional, coletiva e individualmente.

\footnotetext{
${ }^{29}$ ARGENTINA. Ministerio de Salud. Resolución 1083/2009. Disponível em: <http://test.e-legis-ar.msal.gov. ar/leisref/public/showAct.php?mode=PRINT_VERSION\&id=14642>. Acesso em 19 ago. 2014.

${ }^{30}$ Id. Ibid.
} 


\section{Brasil}

No Brasil, a Lei n. 6.259/1975 31 dispõe sobre imunização e doenças de notificação compulsória. Trata-se de uma lei geral e, ainda que antiga, apresenta grande importância para a epidemiologia no país.

Além das normas gerais, existem outros mecanismos para o controle e a prevenção das DCNT. O Decreto n. 5.658/2006 ${ }^{32}$ adota a Convenção de Controle do Uso do Tabaco (OMS), denotando a importância de intervenção necessária sobre o tabaco para que se consiga reduzir as mortes decorrentes das DCNT.

Com o Pacto pela Saúde (Portaria n. 399/2006 33), o Ministério da Saúde estabelece um acordo entre os Conselhos de Saúde e as secretarias municipais de Saúde, definindo responsabilidades nos três níveis (municipal, estadual e federal). Ele é dividido em três grandes componentes, sendo um deles o Pacto pela Vida, que estabelece o cuidado integral de doenças crônicas não transmissíveis como foco prioritário. As principais linhas de ação são: a promoção da saúde, o fortalecimento da atenção básica, a saúde dos idosos e o controle de câncer ${ }^{34}$.

Entre outras ações e dada a importância do tema, o Ministério da Saúde criou o Departamento de Vigilância de Doenças e Agravos Não Transmissíveis e Promoção da Saúde do Ministério da Saúde (DEVDANTPS/SVS/MS), que conta com o apoio do Comitê Gestor da Política Nacional de Promoção da Saúde, da Organização Pan-Americana de Saúde, em sua atuação.

Existem várias políticas nacionais que impactam o controle e a prevenção das doenças crônicas, como exemplo: Política Nacional de Saúde da Pessoa Idosa (PNDPI), disposta na Portaria n. 2.528/2006 35 ; Política Nacional de Promoção da Saúde (PNPS), aprovada conforme a Portaria n. $687 / 2006^{36}$, que está em processo de

${ }^{31}$ BRASIL. Lei n. 6.259, de 30 de outubro de 1975. Dispõe sobre a organização das ações de Vigilância Epidemiológica, sobre o Programa Nacional de Imunizações, estabelece normas relativas à notificação compulsória de doenças, e dá outras providências. Disponível em: <http://www.planalto.gov.br/ccivil_03/ leis/16259.htm>. Acesso em: 30 set. 2014.

${ }^{32}$ BRASIL. Decreto n. 5.658, de 2 de janeiro de 2006. Promulga a Convenção-Quadro sobre Controle do Uso do Tabaco, adotada pelos países membros da Organização Mundial de Saúde em 21 de maio de 2003 e assinada pelo Brasil em 16 de junho de 2003. Disponível em: <http://www.planalto.gov.br/ccivil_03/_ Ato2004-2006/2006/Decreto/D5658.htm>. Acesso em: 30 set. 2014.

${ }^{33}$ MINISTÉRIO DA SAÚDE. Portaria n. 399/GM, de 22 de fevereiro de 2006. Divulga o Pacto pela Saúde 2006 - Consolidação do SUS e aprova as Diretrizes Operacionais do Referido Pacto. Disponível em: <http:// dtr2001.saude.gov.br/sas/PORTARIAS/Port2006/GM/GM-399.htm>. Acesso em: 30 set. 2014.

${ }^{34}$ MINISTÉRIO DA SAÚDE. Instituto Nacional de Câncer José Alencar Gomes da Silva. Saber saúde: prevenção do tabagismo e outros fatores de risco para o desenvolvimento de doenças crônicas não transmissíveis. 3. ed. rev. atual. Rio de Janeiro: INCA, 2013. Disponível em: <http://bvsms.saude.gov.br/bvs/publicacoes/ inca/Saber_saude_2013.pdf>. Acesso em: 29 ago. 2014.

${ }^{35}$ MINISTÉRIO DA SAÚDE. Portaria n. 2.528, de 19 de outubro de 2006. Aprova a Política Nacional de Saúde da Pessoa Idosa. Disponível em: <http://dtr2001.saude.gov.br/sas/PORTARIAS/Port2006/GM/GM-2528. htm>. Acesso em: 30 set. 2014.

${ }^{36}$ MINISTÉRIO DA SAÚDE. Portaria n. 687, de 30 de março de 2006. Aprova a Política de Promoção da Saúde. Disponível em: <http://189.28.128.100/dab/docs/legislacao/portaria687_30_03_06.pdf>. Acesso em: 30 set. 2014. 
revisão ${ }^{37}$; Política Nacional de Alimentação e Nutrição (PNAN), definida na Portaria n. $710 / 1999$ e atualizada conforme a Portaria n 2.715/2011 $1^{38}$.

Por exemplo, a revisão da PNPS tem como objetivo: "promover a equidade e melhoria das condições e modos de viver, ampliando a potencialidade da saúde individual e coletiva, reduzindo vulnerabilidades e riscos à saúde decorrentes dos determinantes sociais, econômicos, políticos, culturais e ambientais". Ainda, o controle do uso do tabaco faz parte da Estratégia Global em Alimentação Saudável, Atividade Física e Saúde, que foi aprovada pela Resolução 57.17 da Assembleia Mundial da Saúde em $2004^{39}$.

Igualmente, a $\mathrm{PNAN}^{40}$, aponta em sua introdução:

O aumento da obesidade está fortemente ligado ao consumo alimentar e à prática de atividade física. Seus determinantes são de natureza demográfica, socioeconômica, epidemiológica e cultural, além de questões ambientais, o que torna a obesidade uma doença multifatorial. Esses fatores interagem de forma complexa, exigindo que a obesidade seja tratada, tendo em vista toda a sua complexidade e determinação social. O Estudo de Carga Global de Doenças no Brasil revela que 58\% dos anos de vida perdidos precocemente se devem às doenças crônicas não transmissíveis.

Essa política pública é central no enfrentamento das DCNT porque a alimentação inadequada é uma dentre as várias causas de desenvolvimento dessas doenças.

\section{Colômbia}

Na Colômbia, as DCNT representam a maior carga de morbimortalidade; em 2008 a prevalência de hipertensão arterial em adultos foi de 8,8\% e a de diabetes chegou a $3,5 \%$. As doenças cardiovasculares foram a primeira causa de morte na população, representando 42,6 em cada 100 mil habitantes ${ }^{41}$.

\footnotetext{
${ }^{37}$ Atualmente estm emualmente es apreciante CITeciante estm: encaminhada para a "Apreciasms.saude.g e finalmente posterior publica.saude.gov.br/bv

${ }^{38}$ MINISTÉRIO DA SAÚDE. Portaria n. 2.715, de 17 de novembro de 2011. Atualiza a Política Nacional de Alimentação e Nutrição. Disponível em: <http://crn3.org.br/legislacao/doc/Portaria_MS_\%20 2715-2011-atualiza_PNAN.pdf>. Acesso em: 30 set. 2014.

${ }^{39}$ ORGANIZAÇÃO PAN-AMERICANA DE SAÚDE (OPAS). WHA57.17. Global strategy on diet, physical activity and health. Disponivel em: <http://www.paho.org/bra/index.php?option=com_docman\&task=doc_ view\&gid=1181\&ltemid=614>. Acesso em: 03 set. 2014.

${ }^{40}$ MINISTÉRIO DA SAÚDE. Secretaria de Atenção a Saúde, Departamento de Atenção Básica. Política Nacional de Alimentação e Nutrição. Brasília/DF, 2012. p. 19. Disponível em: <http://189.28.128.100/nutricao/docs/geral/pnan2011.pdf>. Acesso em: 29 ago. 2014.

${ }^{41}$ ORGANIZAÇÃO PAN-AMERICANA DA SAÚDE (OPAS). Saúde nas Américas: panorama geral e perfis de países, 2012, cit.
} 
Em período semelhante ao dos demais países latino-americanos, também nos anos 2000, especificamente em 2007, que foi elaborado um Plan Nacional de Salud Pública para los años de 2007 hasta 2010, disposto no Decreto n. 3.039 de 10 de agosto de $2007^{42}$ :

Decreto n. 3.039, de 10 de agosto de 2007, adota o Plano Nacional de Saúde Pública 2007-2010.

Meta 6.

Reduzir Doenças Não Transmissíveis e Deficiência.

As metas nacionais para as doenças não transmissíveis e incapacidades.

1. Aumentar acima de $26 \%$ a prevalência de atividade física em adolescentes entre 13 e 17 anos (linha de base: 26\% Fonte: ENSIN 2005).

2. Aumentar acima de $42,6 \%$ à prevalência de atividade física em adultos com idade entre 18 a 64 anos (linha de base: $42,6 \%$ Fonte: ENSIN 2005).

3. Aumentar acima da média de 12,7 anos o início do consumo de cigarros na população com menos de 18 anos (linha de base: 12,7 anos Fonte: Pesquisa Nacional de Drogas, escola 2004).

4. Promover as ações de diagnóstico precoce da Doença Renal Crônica - DRC (linha de base: a ser determinada).

5. Promover ações preventivas para manter ou reduzir a prevalência de limitações evitáveis (linha de base: 6,4\% Fonte: DANE 2005).

Em 2012, contando com a participação de grande parcela da população, foi promulgado o Plan Decenal de Salud Pública 2012-2021 ${ }^{43}$, que tem como principal objetivo:

alcançar a equidade em saúde e desenvolvimento humano de todos os colombianos e colombianas mediante oito dimensões prioritárias e duas transversais, que representam os aspectos fundamentais em que, por sua magnitude ou importância, se deve intervir, preservar ou melhorar, para garantir a saúde e o bem-estar de todos os colombianos, sem distinção de gênero, etnia, ciclo de vida, nível socioeconômico ou qualquer outra situação diferencial.

\footnotetext{
${ }^{42}$ ORGANIZAÇÃO PAN-AMERICANA DA SAÚDE (OPAS). Recopilación de normas sobre prevención y control de enfermedades crónicas en América Latina: obesidad, diabetes y enfermedades cardiovasculares. Washington, D.C., nov. 2009. p. 61-63.

${ }^{43}$ COLOMBIA. Resolución n. 1841, de 28 de maio de 2013, por a cual se adopta el Plan Decenal de Salud Pública 2012-2021. Disponível em: <http://www.minsalud.gov.co/sites/rid/Lists/BibliotecaDigital/RIDE/ DE/DIJ/Resoluci\%C3\%B3n\%201841\%20de\%202013.pdf>. Acesso em: 08 set. 2014.
} 
O Plan Decenal 2012-2021 apresenta muitos dados epidemiológicos em sua justificativa, o que denota não só um estudo aprofundado das principais questões de saúde pública, como também as possíveis formas para diminuir esses agravos.

Associada às doenças crônicas não transmissíveis, a prevalência de fatores de risco para a doença e a morte de maior peso entre a população colombiana são: tabagismo, má alimentação e falta de atividade física; além da presença de condições ambientais relativas a qualidade do ar, estresse urbano, infecções e exposições ocupacionais (Ministério da Saúde e Proteção Social, Instituto Nacional do Câncer, ESE, 2012) ${ }^{44}$.

As DCNT na Colômbia representam um grave problema de saúde pública e, como nos demais países, a legislação nos indica que um grande trabalho precisa ser realizado. Assim, contar com a participação da população na elaboração das normas é fundamental para o sucesso dessa empreitada em prol da redução das DCNT.

\section{Considerações finais}

Em razão de sua magnitude, as DCNT representam atualmente uma questão de saúde que ultrapassou os limites geográficos e de renda: são um tema de interesse global. Organizações como a OMS estão voltadas a oferecer modelos de políticas públicas, scripts para que os países possam acessá-la se adequá-las a sua realidade e, assim sejam capazes de enfrentar essa situação com algum sucesso.

Alguns planos foram traçados em prol da redução das DCNT no mundo, em geral orquestrados pela OMS. A realidade é que atualmente o número de vidas que se perdem em razão das DCNT é alto e, entre as principais causas, estão as doenças cardiovasculares.

Tratar conjuntamente das DCNT é o melhor caminho para o sucesso. Os fatores de risco conjugados apontam para políticas públicas amplas que terão como principal característica a redução do número de mortes decorrentes das DCNT. Otimizar os esforços e informar a população, bem como atuar regulamentando a indústria alimentícia e criar espaços para a prática de atividades físicas são o grande foco das políticas existentes nos países latino-americanos.

Ademais, sendo a obesidade um dos principais fatores de risco entre as DCNT, políticas públicas de incentivo ao pequeno produtor para aumentar a qualidade das frutas, verduras e legumes consumidos trariam importante mudança nos hábitos alimentares da população, especialmente nos países de baixa e média renda.

\footnotetext{
${ }^{44}$ COLOMBIA. Resolución n. 1841, de 28 de maio de 2013, por a cual se adopta el Plan Decenal de Salud Pública 2012-2021, cit., p. 76.
} 
As mortes decorrentes das DCNT exigem que governos, população e indivíduos atuem de forma concentrada: reduzir os fatores de risco e, assim, diminuir o número de pessoas com DCNT tem que ser um objetivo comum, em que todos deverão atuar dentro de suas possibilidades para que o sucesso seja alcançado.

\section{Referências}

ARGENTINA. Poder Ejecutivo Naciónal. Plan Naciónal de Abordaje Integral. Disponível em: $<$ http://test.e-legis-ar.msal.gov.ar/leisref/public/showAct.php?id=7444\&word=>. Acesso em: 18 ago. 2014.

BEAGHOLE, R. et al. NCD Countdown 2025: accountability for the $25 \times 25$ NCD mortality reduction target. The Lancet, v. 384, n. 9938, p. 105-107, 2014. Disponível em: <http://www. thelancet.com/pdfs/journals/lancet/PIIS0140673614610916.pdf>. Acesso em: 13 set. 2014.

et al. Priority actions for the non-communicable disease crisis. The Lancet, v. 377, p. 1438-1447, 2011.

BLAIR, S. N.; DANIELS, S. R.; ECKEL, R. H.; FAIR, J. M. et al. AHA guidelines for primary prevention of cardiovascular disease and stroke: 2002 update: Consensus panel guide to comprehensive risk reduction for adult patients without coronary or other atherosclerotic vascular diseases. Circulation, v. 106, n. 3, p. 388-391, 2002.

BUCCI, M. P. Dallari. O conceito de política pública em direito. In: BUCCI, M. P. Dallari (Org.). Políticas públicas: reflexões para um conceito jurídico. São Paulo: Saraiva; Santos/SP: UNISANTOS, 2006.

CAMPOS, T. et al. Cigarette smoking among patients with chronic diseases. International Journal of Cardiology, v. 174, n. 3, p. 808-810, 2014.

CHIOLERO, R.; FAEH, D.; PACCAUD, F.; CORNUZ, J. Consequences of smoking for body weight, body fat distribution, and insulin resistance. American Society for Clinical Nutriton, v. 87, n. 4 , p. $801-809,2008$.

CHRONIC diseases and development. High-level Meeting of the General Assembly on chronic non-communicable diseases. The Lancet. Disponível em: <http://www.thelancet.com/series/ chronic-diseases-and-development>. Acesso em: 13 set. 2014.

DICIONÁRIO do Coração - Termos e Procedimentos. Disponível em: <http://prevencao. cardiol.br/biblioteca/principal.asp\#D>. Acesso em: 06 set. 2014.

FRIEDMAN, R. R.; SCHWARTZ, M. B. Public policy to prevent childhood obesity and the role of pediatric endocrinologists. Journal of Pediatric Endocrinology \& Metabolism, v. 21, p. 717-725, 2008. 
GORDON, R.; McDERMOTT, L.; STEAD, M.; ANGUS, K. The effective ness of social marketing interventions for health improvement: what's the evidence? Public Health, v. 120, n. 12, p. 1133-1139, 2006.

JEWELL, J.; HAWKES, C.; ALLEN, K. Law and obesity prevention: addressing some key questions for the public health community. WCRF International, 2013.

LANG, T.; RAYNER, G. Over coming policy cacophonyon obesity: na ecological public health framework for policymakers. Obesity Reviews, v. 8, p. 165-181, 2007.

MAGNUSSONR. S. What's law gotto do with it? Part I: a framework for obesity prevention. Australian and New Zealand Health Policy, v. 5, p. 1-12, 2008.

MARTIN, R. The role of law in the control of obesity in England: looking at the contribution of law to a healthy food culture. Australia and New Zealand Health Policy, v. 5, n. 21, 2008. Disponível em: <http://www.ncbi.nlm.nih.gov/pmc/articles/PMC2615029/>. Acesso em: 26 out. 2014.

MINISTÉRIO DA SAÚDE. Instituto Nacional de Câncer José Alencar Gomes da Silva. Saber saúde: prevenção do tabagismo e outros fatores de risco para o desenvolvimento de doenças crônicas não transmissíveis. 3. ed. rev. atual. Rio de Janeiro: INCA, 2013. Disponível em: $<$ http:// bvsms.saude.gov.br/bvs/publicacoes/inca/Saber_saude_2013.pdf>. Acesso em: 29 ago. 2014.

. Secretaria de Atenção a Saúde, Departamento de Atenção Básica. Política Nacional de Alimentação e Nutrição. Brasília/DF, 2012. Disponível em: <http://189.28.128.100/nutricao/ docs/geral/pnan2011.pdf>. Acesso em: 29 ago. 2014.

ORGANIZAÇÃO MUNDIAL DA SAÚDE (OMS). O Dia Mundial do Coração 2013 preconiza hábitos de vida saudável desde a infância. Disponível em: <http://www.paho.org/bireme/ index.php?id=219\%3Ao-dia-mundial-do-coracao-2013-preconiza-habitos-de-vida-saudaveldesde-a-infancia\&option=com_content $>$. Acesso: em 07 set. 2014.

Diabetes Mellitus. Fact Sheets, n. 312, Oct. 2013. Disponível em: <http://www.who. int/mediacentre/factsheets/fs312/en/index.html>. Acesso em: 23 ago. 2014.

. Doenças Cardiovasculares. Fact Sheets, n. 317, Mar. 2013. Disponível em: <http:// www.who.int/mediacentre/factsheets/fs317/en/index.html>. Acesso em: 31 ago. 2014.

Obesity and overweight. Fact Sheets, n. 311, Aug. 2014. Disponível em: <http://www. who.int/mediacentre/factsheets/fs311/en/index.html>. Acesso em: 01 set. 2014.

ORGANIZAÇÃO PAN-AMERICANA DA SAÚDE (OPAS). Recopilación de normas sobre prevención y control de enfermedades crónicas en América Latina: obesidad, diabetes y enfermedades cardiovasculares. Washington, D.C., nov. 2009.

Saúde nas Américas: panorama geral e perfis de países, 2012. Disponível em: <http:// apsredes.org/site2012/wp-content/uploads/2012/09/Sa\%C3\%BAde-nas-Am\%C3\%A9ricas3. pdf $>$. Acesso em: 28 ago. 2014. 
PAN AMERICAN HEALTH ORGANIZATION. Population and individual approaches to the prevention and management of diabetes and obesity. Disponível em: $<$ www.paho.org/hq/index. php?option=com_docman\&task=doc_view\&gid=15557\&Itemid=>. Acesso em: 26 out. 2014.

POMERANZ, J. L.; GOSTIN, J. D. Improving laws and legal authorities for obesity prevention and control. Journal of Law, Medicine and Ethics, n. 37, p. 62-75, 2009.

SRINIVASAN, S.; O'FALLON, L. R.; DEARRY, A. Creating healthy communities, healthy homes, healthy people: research agenda on the built environment and public health. American Journal of Public Health, v. 93, n. 9, p. 1446-1450, 2003.

SWINBURN B.; EGGER, G. Preventive Strategy against Weight Gain and Obesity. Obesity Reviews, v. 3, p. 289-301, 2002.

Rachelle Amália Agostini Balbinot - Graduada em Direito pela Universidade Federal de Santa Maria; Mestre em Direito pela Universidade Federal de Santa Catarina; Doutora em Integração da América Latina pela Universidade de São Paulo; PósDoutora em Saúde Pública pela Faculdade de Saúde Pública da Universidade de São Paulo. Vice-Diretora e pesquisadora do Centro de Estudos e Pesquisas em Direito Sanitário; Pesquisadora doNúcleo de Pesquisa em Direito Sanitário da Universidade de São Paulo. Advogada. São Paulo/SP, Brasil. E-mail: rachelle@usp.br. 